\section{Validation of the International Prognostic Index and Subsequent Revisions for Diffuse Large B-Cell Lymphoma in Patients From the Middle East and North Africa Region}

Faisal Alamer ${ }^{1}$, Ahmad Alamir ${ }^{1}$, Abdulrahman Alqahtani ${ }^{1}$, Abdulrahman M. Alkabli ${ }^{1}$, Homod Alshabib ${ }^{1}$ , Moussab Damlaj ${ }^{1,2,3}$

1. Oncology, King Abdulaziz Medical City, Riyadh, SAU 2. Medicine, King Abdullah International Medical Research Center, Riyadh, SAU 3. Medicine, King Saud Bin Abdulaziz University for Health Sciences, Riyadh, SAU

Corresponding author: Moussab Damlaj, moussab.damlaj@mail.mcgill.ca
Received 07/08/2020 Review began $07 / 11 / 2020$ Review ended 07/23/2020 Published 08/09/2020

๑) Copyright 2020 Alamer et al. This is an open access article distributed under the terms of the Creative Commons Attribution License CC-BY 4.0., which permits unrestricted use, distribution, and reproduction in any medium, provided the original author and source are credited.

\begin{abstract}
Diffuse large B-cell lymphoma (DLBCL) is a heterogenous disease with a variable prognosis. The International Prognostic Index (IPI), revised-IPI (R-IPI), and National Comprehensive Cancer Network-IPI (NCCN-IPI) have been developed and validated to predict prognosis in DLBCL. However, patients from the Middle East and North Africa (MENA) region were under-represented in such scores, and it is unclear whether ethnic background contributes to different disease biology or response to therapy. Following due Institutional Board Review approval, DLBCL patients diagnosed from January 2010 until December 2015 from the MENA region were retrospectively reviewed. A total of 122 were identified and further analyzed. There were 74 males (61\%), and the median age at diagnosis for the cohort was 64 years (range: 18-98 years), with a median follow-up duration of 32.9 months (range: 0.2-123.7 months). Estimates of three-year progression-free survival found a significant difference among risk groups using all three prognostic models but were more discriminating among the groups using NCCN-IPI and R-IPI vs. IPI ( $p=0.019$ and 0.014 vs. 0.039 , respectively). For overall survival estimates at three years, the NCCN-IPI was the best model compared to R-IPI and IPI ( $p=0.0013$ vs. 0.05 and 0.04 , respectively). In conclusion, we validated that the IPI and its subsequent iterations were predictive of outcome in DLBCL patients from the MENA region; however, the NCCN-IPI appeared the most prognostic. These results warrant further confirmation.
\end{abstract}

Categories: Oncology, Hematology

Keywords: duodenal diffuse large b-cell lymphoma, international prognostic index, middle east north africa region

\section{Introduction}

Diffuse large B-cell lymphoma (DLBCL) is a commonly diagnosed non-Hodgkin lymphoma (NHL), accounting for one-quarter of NHL cases [1]. Approximately, half of the patients afflicted with DLBCL can be cured when treated with chemo-immunotherapy approaches typically consisting of combinational chemotherapy combined with the CD20 monoclonal antibody rituximab [2]. In Saudi Arabia, NHL is the third most commonly diagnosed malignancy with an incidence rate of $6 / 100,000$, with the most prevalent NHL subtype being DLBCL [3].

DLBCL remains a heterogeneous disease with differing presentations and clinical outcome. Therefore, the identification of prognostic tools is of paramount importance to aid physicians to more accurately riskstratify their patients. The International Prognostic Index (IPI) was originally developed in order to assess the impact of pre-treatment factors on outcome. The identified factors included age $>60$ years, lactate dehydrogenase level (LDH) above normal, Eastern Cooperative Oncology Group (ECOG) performance status $>2$, stage III or IV disease, and the presence of more than one extra-nodal site [4]. Cumulative risks are tallied to estimate the risk status in one of four categories, low, low-intermediate, high-intermediate, or high-risk disease, with significantly differing overall survival (OS) and relapse-free survival (RFS) among the groups.

As the original IPI was derived from cohorts that have not received rituximab, subsequent efforts were undertaken to revise this score in contemporarily treated patients. Sehn et al. undertook such an effort and the reported revised IPI (R-IPI) identified three prognostic groups - very good, good, and poor - again with differing outcomes [5]. Finally, the National Comprehensive Cancer Network (NCCN) did further refinement of the score to include age and LDH as continuous variables and the location instead of the number of extranodal lesions [6]. Using the NCCN-IPI, patients were again classified into four groups similar to the original IPI, i.e., low, low-intermediate, high-intermediate, or high risk.

Importantly, patients from the Middle East and North Africa (MENA) region were under-represented in the derivation of such prognostic scores. This raises important questions on whether the IPI and subsequent versions can accurately predict prognosis in this ethnic population. For example, an effort to validate the 
original IPI in a Chinese population reported that the presence of extra-nodal disease was not an important element in the score to stratify patients [7]. The aim from of analysis is to validate the original IPI and its subsequent iterations in a Saudi cohort from the MENA region.

\section{Materials And Methods Study design and patients}

This was a retrospective single-center study conducted at King Abdulaziz Medical City, Riyadh, Saudi Arabia, following Institutional Board Review approval. Patients $\geqslant 14$ years of age with a diagnosis of DLBCL from January 2010 until December 2015 were identified and data were retrieved through a query of the institutional Oncology database. Diagnosis of DLBCL was per the World Health Organization 2016 Classification and based on a combination of compatible morphology, immunophenotype, and genetic profile [8]. Clinical and pathologic variables were retrospectively abstracted. Majority of patients were treated with combinational chemotherapy containing rituximab. Patients who died prior to receiving therapy were excluded.

\section{Computation of the IPI and subsequent revised versions}

The IPI was calculated based on the following variables as previously described, with one point being assigned to each: age 60 years and older, elevated LDH level, stage III-IV disease, ECOG score, and more than one extra-nodal site of disease [4]. The total score was tallied and patients were stratified into different risk groups as follows: low with a score of 0 -1, low-intermediate with a score of 2 , high-intermediate with a score of 3, and high with a score of 4-5.

The R-IPI was computed using the same variables as the original IPI but with only the following three distinct prognostic groups: very good with a score of 0 , good with a score of $1-2$, and poor with a score of 3-5 [5]. Finally, the NCCN-IPI was computed by using the same variables but with further refinement of age into groups ( $\leqslant 40,41-60,61-75$, and $>75$ ), LDH (normal, $\leqslant 3 x$ upper limit of normal, or $>3 x$ upper limit of normal), and specifying the location of extra-nodal sites to bone marrow, central nervous system, liver, gastrointestinal tract, or lung, whereas ECOG and stage remained the same. Using the NCCN-IPI, patients were again classified into four groups similar to the original IPI, i.e., low with a score of 0-1, lowintermediate with a score of 2-3, high-intermediate with a score of 4-5, or high risk with a score of 6-8 [6].

\section{Definitions and statistical analysis}

All variables were collected retrospectively with baseline patient, disease, and treatment characteristics reported as frequency, median, and/or percentages. Comparisons between variables were made using the Pearson and Wilcoxon/Kruskal-Wallis tests for categorical and continuous variables as appropriate. The Kaplan-Meier method was used to estimate progression-free survival (PFS), and OS and was reported as a percentage with log-rank test for group comparison. The definition of OS was the time from the diagnosis of DLBCL until the date of death due to any factor or last documented follow-up. Relapse, progression, or death was considered an event for PFS estimation. JMP Pro Version 11 software (SAS Institute, Cary, NC, USA) was used for statistical analysis.

\section{Results}

\section{Patient characteristics}

A total of 122 patients were identified and further analyzed. There were 74 males (61\%), and the median age at diagnosis for the cohort was 64 years (range: 18-98 years). Presenting median counts were as follows: white blood count of 7 x 109/L (normal range: 0.7-183 x 109/L), hemoglobin of $112 \mathrm{~g} / \mathrm{L}$ (normal range: 67170), and platelets of $295 \times 109 / \mathrm{L}$ (normal range: $29-819 \times 109 / \mathrm{L}$ ). A total of 69 patients (57\%) were over the age of 60 years. LDH was elevated in 91 (75\%) of patients. The median ECOG was 1 (0-4). There were 97 (80\%) patients with stage III or IV disease, and 41 (34\%) of patients had extra-nodal disease. The median follow-up duration was 32.9 months (range: 0.2-123.7 months) during which a total of 28 (23\%) patients experienced disease relapse or progression and 44 (36\%) died. The median follow-up of alive patients was 40.2 months. The baseline characteristics of patients are shown in Table 1.

\section{Characteristics}

Age, median (range)

Gender, n (\%)

Male

Female

Stage, n (\%)

\section{N (\%)}

$64(18-98)$

48 (39) 


\section{Cureus}

\section{I/II}

$25(20 \%)$

III/IV

97 (80\%)

ECOG, median (range)

$1(0-4)$

Elevated LDH, $\mathrm{n}(\%)$

$91(75 \%)$

Extra-Nodal Disease, $\mathrm{n}(\%)$

41 (34\%)

IPI, n (\%)

Low

Low-Intermediate

High-Intermediate

32 (26)

High

R-IPI, n (\%)

Very Good

3 (2)

Good

Poor

NCCN-IPI, n (\%)

Low

8 (6)

Low-Intermediate

41 (34)

High-Intermediate

51 (42)

High

Chemotherapy Used, n (\%)

R-CHOP

R-CHOP/R-CVP

R-CVP

Palliative

Not Treated

IFRT, $\mathrm{n}(\%)$

End of Treatment Response, $n$ (\%)

Complete Response

$76(62 \%)$

Partial Response

Progressive Disease

$15(12 \%)$

N/A

$18(15 \%)$

Follow-up months, median (range)

$32.9(0.2-123.7)$

\section{TABLE 1: Baseline characteristics of the cohort}

ECOG, eastern cooperative oncology group; LDH, lactate dehydrogenase; IPI, International Prognostic Index; R-IPI, revised-IPI; NCCN-IPI, National Comprehensive Cancer Network-IPI; R-CHOP, rituximab, cyclophosphamide, doxorubicin, vincristine, and prednisone; R-CVP, rituximab with cyclophosphamide, vincristine, and prednisone; IFRT, involved field radiotherapy; N/A, not available

\section{Treatment received and response assessment}

Standard frontline therapy was combinational chemotherapy with cyclophosphamide, vincristine, doxorubicin, and prednisone with the monoclonal antibody rituximab (R-CHOP) [9]. This treatment protocol was modified in unfit or elderly patients at the discretion of the treating physician to reduce or omit 
doxorubicin while maintaining a curative approach of therapy. An additional group of patients were deemed unfit for systemic therapy and were given palliative approach to treatment including supportive measures only.

The breakdown of therapy was as follows: the majority of patients received standard R-CHOP at 88 (72\%), an additional 18 (15\%) received modified R-CHOP, 3 (2\%) received R-CVP (rituximab with cyclophosphamide, vincristine, and prednisone), 5 (4\%) received palliative rituximab with or without oral chemotherapy, and the remaining 8 (7\%) did not receive any systemic therapy and were managed symptomatically. Additionally, 33 (27\%) patients received involved field radiotherapy. End of treatment response was as follows: complete response (CR) in 76 (62\%), partial response in 13 (11\%), progressive disease in 15 (12\%), and the remaining 18 (15\%) did not have an end of treatment evaluation due to being in the palliative therapy group or early death due to sepsis/multi-organ failure while on active curative intent therapy.

\section{Outcome stratified by IPI}

\section{Original IPI}

Stratified according to the different risk groups, a total of 20 (16\%), 30 (25\%), 32 (26\%), and 40 (33\%) were scored as low, low-intermediate, high-intermediate, and high, respectively. The corresponding three-year OS ( $\pm 95 \%$ confidence interval) for patients with low, low-intermediate, high-intermediate, and high risk was $84 \% \pm 8,75.8 \% \pm 8,67 \% \pm 9$ and $51.1 \% \pm 8$, respectively $(\mathrm{p}=0.04)$. On the other hand, the three-year PFS ( $\pm 95 \%$ confidence interval) for patients with low, low-intermediate, high-intermediate, and high risk was $78.8 \% \pm 9,69 \% \pm 9,45.7 \% \pm 9$ and $40.4 \%, \pm 8$, respectively $(\mathrm{p}=0.039$ ), as shown in Figures $1 \mathrm{~A}, 1 \mathrm{~B}$ and Table 2 .

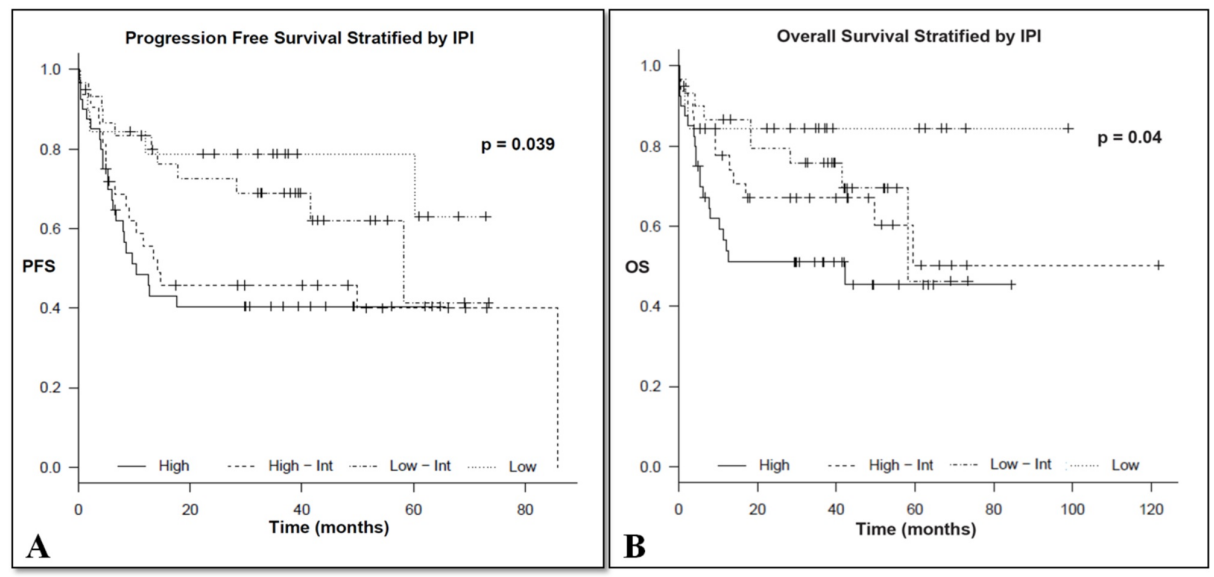

FIGURE 1: Progression free survival (A) and overall survival (B) stratified by IPI

IPI, International Prognostic Index 


\section{Cureus}

\begin{tabular}{|c|c|c|c|}
\hline Risk group & Score & Three-year PFS, \% & Three-year OS, \% \\
\hline \multicolumn{4}{|l|}{ Original IPI } \\
\hline Low & $0-1$ & $78.8 \% \pm 9$ & $84 \% \pm 8$ \\
\hline Low-Intermediate & 2 & $69 \% \pm 9$ & $75.8 \% \pm 8$ \\
\hline High-Intermediate & 3 & $45.7 \% \pm 9$ & $67 \% \pm 9$ \\
\hline High & $4-5$ & $40.4 \% \pm 8$ & $51.1 \% \pm 8$ \\
\hline \multicolumn{4}{|l|}{ Revised IPI } \\
\hline Very Good & 0 & $100 \%$ & $100 \%$ \\
\hline Good & $1-2$ & $70.8 \% \pm 7$ & $77.6 \% \pm 6$ \\
\hline Poor & $3-5$ & $42.7 \% \pm 6$ & $58 \% \pm 6$ \\
\hline \multicolumn{4}{|l|}{ NCCN-IPI } \\
\hline Low & $0-1$ & $83.3 \% \pm 15$ & $100 \%$ \\
\hline Low-Intermediate & $2-3$ & $67.4 \% \pm 7$ & $79.6 \% \pm 6$ \\
\hline High-Intermediate & $4-5$ & $49.6 \% \pm 7$ & $63.5 \% \pm 7$ \\
\hline High & 6 or above & $34.3 \% \pm 11$ & $39.2 \% \pm 11$ \\
\hline
\end{tabular}

\section{TABLE 2: Outcome stratified according to different IPI scores}

IPI, International Prognostic Index; NCCN-IPI, National Comprehensive Cancer Network-IPI

\section{Revised IPI}

Stratified according to the different risk groups, a total of 3 (2\%), 47 (39\%), and 72 (59\%) were scored as very good, good, and poor, respectively. The corresponding three-year OS ( $\pm 95 \%$ confidence interval) for patients with very good, good, and poor risk was $100 \%, 77.6 \% \pm 6$, and $58 \% \pm 6$, respectively $(\mathrm{p}=0.05)$. On the other hand, the three-year PFS ( $\pm 95 \%$ confidence interval) for patients with very good, good, and poor risk was $100 \%, 70.8 \% \pm 7$ and $42.7 \% \pm 6$, respectively $(\mathrm{p}=0.014)$, as shown in Figures $2 A, 2 B$ and Table 2 .

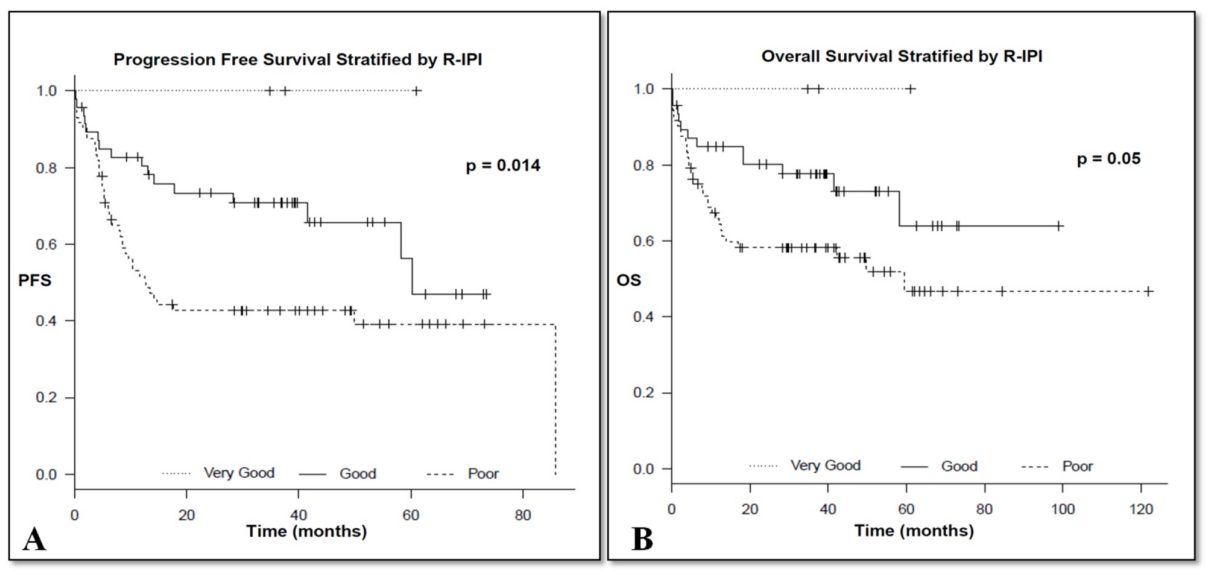

FIGURE 2: Progression free survival (A) and overall survival (B) stratified by R-IPI

R-IPI, revised International Prognostic Index

\section{NCCN-IPI}

Stratified according to the different risk groups, a total of 8 (6\%), 41 (34\%), 51 (42\%), and 22 (18\%) were 
scored as low, low-intermediate, high-intermediate, and high, respectively. The corresponding three-year OS ( $\pm 95 \%$ confidence interval) for patients with low, low-intermediate, high-intermediate, and high risk was $100 \%, 79.6 \% \pm 6,63.5 \% \pm 7$, and $39.2 \% \pm 11$, respectively $(\mathrm{p}=0.0013)$. On the other hand, the three-year PFS ( $\pm 95 \%$ confidence interval) for patients with low, low-intermediate, high-intermediate, and high risk was $83.3 \% \pm 15,67.4 \% \pm 7,49.6 \% \pm 7$, and $34.3 \% \pm 11$, respectively $(\mathrm{p}=0.019)$, as shown in Figures $3 A, 3 B$ and Table 2 .
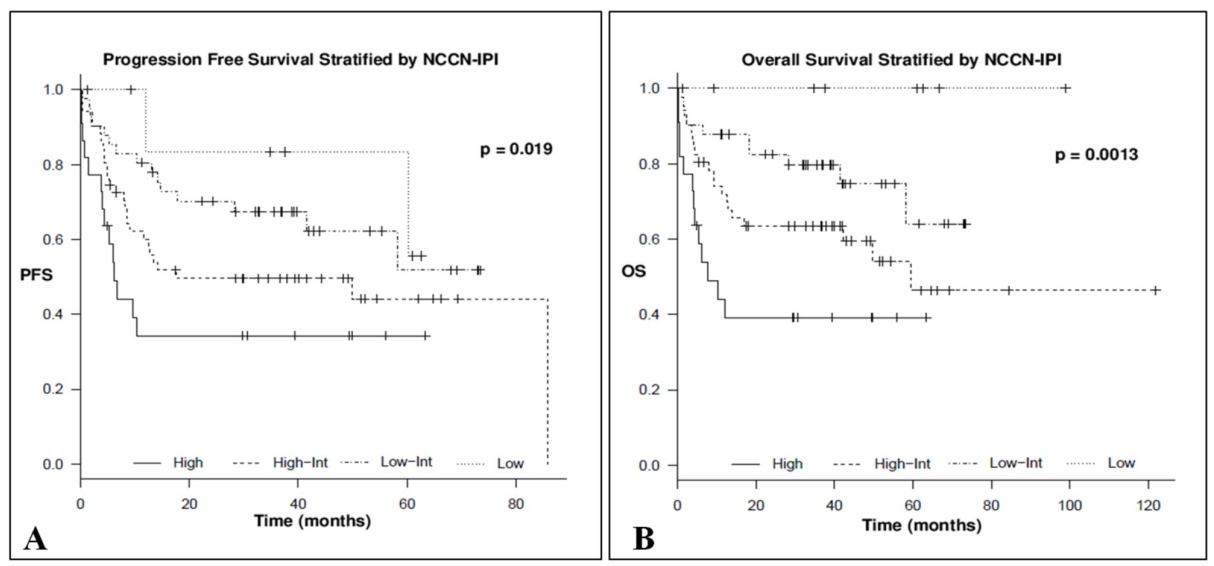

\section{FIGURE 3: Progression free survival (A) and overall survival (B) stratified by NCCN-IPI}

NCCN-IPI, National Comprehensive Cancer Network International Prognostic Index

\section{Discussion}

DLBCL is a common lymphoma representing approximately $25 \%$ of all NHL cases. However, it is a heterogeneous disease morphologically, genetically, and even biologically [8]. It is curable in approximately half of the patients, particularly in those who can attain a complete response. The IPI was proposed as a prognostic tool since 1993 to predict OS and RFS in aggressive NHL patients who receive combinational chemotherapy containing doxorubicin. The corresponding five-year OS in the initial group of 2,031 patients assigned to low, low-intermediate, high-intermediate, and high-risk groups was $73 \%, 51 \%$, 43\%, and $26 \%$, respectively. Subsequently, with the incorporation of rituximab as part of combinational chemoimmunotherapy, the original IPI was revised into three risk groups that were initially reported by the British Columbia Cancer Agency and later re-examined using data from three prospective trials $[9,10]$. Most recently, the NCCN-IPI was derived using the same variables but with further refinement of LDH and age as continuous variables and considering extra-nodal disease involving bone marrow, central nervous system, liver/gastrointestinal tract, or lung as significant. For the four risk groups, i.e., low, low-intermediate, highintermediate, and high, the resulting five-year OS was $96 \%, 77 \%, 56 \%$, and $38 \%$, respectively, and the PFS was $94 \%, 72 \%, 54 \%$, and $35 \%$, respectively.

Given the heterogeneous nature of DLBCL, it is unclear whether ethnic background contributes to different disease biology or response to therapy. Furthermore, patients from the MENA region were not represented in the above risk scores. Therefore, the aim of this study is to examine whether the IPI and its subsequent derivations can be used to predict outcome in patients from the MENA region. We observed that the proportion of patients with low/very good risk disease is low, which is consistent with prior reports showing a higher percentage of patients in this part of the world presenting with advanced stage or extra-nodal disease [3]. With regard to PFS estimation, although statistically significant, the original IPI did not discriminate well among the four groups specifically between the lower risk groups (low and lowintermediate) and higher risk groups (high-intermediate and high). On the other hand, PFS using R-IPI was more divergent and most significant using the NCCN-IPI. When looking at OS, we again note that the discrimination potential is less profound with the original IPI where the intermediate group (low and high) fare similarly compared with R-IPI and NCCN-IPI. Our findings suggest that the NCCN-IPI is the most useful score to discriminate among the risk groups in MENA patients followed by the R-IPI. This observation was also noted in an Asian cohort of patients where the NCCN-IPI was superior to the original IPI [7].

This analysis is limited by its retrospective nature and sample size. The proportion of patients with low/very good risk disease is lower than previous reports and could be a further limiting factor. Despite these limitations, this report generates additional insight that the NCCN-IPI is the best prognostic tool when evaluating patients from the MENA region. This observation warrants further validation.

\section{Conclusions}


In conclusion, we validated that the IPI and its subsequent iterations were predictive of outcome in DLBCL patients from the MENA region; however, the NCCN-IPI appeared the most prognostic. These results warrant further confirmation.

\section{Additional Information \\ Disclosures}

Human subjects: Consent was obtained by all participants in this study. King Abdullah International Medical Research Center (KAIMRC) issued approval SP17-065. Animal subjects: All authors have confirmed that this study did not involve animal subjects or tissue. Conflicts of interest: In compliance with the ICMJE uniform disclosure form, all authors declare the following: Payment/services info: All authors have declared that no financial support was received from any organization for the submitted work. Financial relationships: All authors have declared that they have no financial relationships at present or within the previous three years with any organizations that might have an interest in the submitted work. Other relationships: All authors have declared that there are no other relationships or activities that could appear to have influenced the submitted work.

\section{References}

1. Morton LM, Wang SS, Devesa SS, Hartge P, Weisenburger DD, Linet MS: Lymphoma incidence patterns by WHO subtype in the United States, 1992-2001. Blood. 2006, 107:265-276. 10.1182/blood-2005-06-2508

2. Jardin F: Improving R-CHOP in diffuse large B-cell lymphoma is still a challenge . Lancet Oncol. 2019, 20:605-606. 10.1016/S1470-2045(19)30021-X

3. Rauf MS, Akhtar S, Maghfoor I: Changing trends of adult lymphoma in the Kingdom of Saudi Arabia comparison of data sources. Asian Pac J Cancer Prev. 2015, 16:2069-2072. 10.7314/apjcp.2015.16.5.2069

4. The International Non-Hodgkin's Lymphoma Prognostic Factors Project: A predictive model for aggressive non-Hodgkin's lymphoma. N Engl J Med. 1993, 329:987-994. 10.1056/NEJM199309303291402

5. Sehn LH, Berry B, Chhanabhai M, et al.: The revised International Prognostic Index (R-IPI) is a better predictor of outcome than the standard IPI for patients with diffuse large B-cell lymphoma treated with RCHOP. Blood. 2007, 109:1857-1861. 10.1182/blood-2006-08-038257

6. Zhou Z, Sehn LH, Rademaker AW, et al.: An enhanced International Prognostic Index (NCCN-IPI) for patients with diffuse large B-cell lymphoma treated in the rituximab era. Blood. 2014, 123:837-842. 10.1182/blood-2013-09-524108

7. Huang CE, Chen YY, Lu CH, Chen PT, Lee KD, Chen CC: Validation of an enhanced International Prognostic Index (NCCN-IPI) in an Asian cohort of patients with diffuse large B cell lymphoma. Ann Hematol. 2015, 94:1063-1065. 10.1007/s00277-014-2293-8

8. Swerdlow SH, Campo E, Pileri SA, et al.: The 2016 revision of the World Health Organization classification of lymphoid neoplasms. Blood. 2016, 127:2375-2390. 10.1182/blood-2016-01-643569

9. Tilly H, Gomes da Silva M, Vitolo U, et al.: Diffuse large B-cell lymphoma (DLBCL): ESMO Clinical Practice Guidelines for diagnosis, treatment and follow-up. Ann Oncol. 2015, 26:116-125. 10.1093/annonc/mdv304

10. Bari A, Marcheselli L, Sacchi S, et al.: Prognostic models for diffuse large B-cell lymphoma in the rituximab era: a never-ending story. Ann Oncol. 2010, 21:1486-1491. 10.1093/annonc/mdp531 